\title{
Estudo da localização de escolas municipais no município de São Jerônimo da Serra - PR
}

\author{
Aline Santos de Araújo, Luzia Vidal de Souza \\ Universidade Federal do Paraná - PPGMNE \\ 81531-970, Campus III, Curitiba, PR \\ E-mails: prof.alinearaujo@gmail.com 는.bufpr.br, \\ Danielle Durski Figueiredo \\ Universidade Tecnológica Federal do Paraná - Departamento de Matemática \\ 80230-901, Campus Curitiba, Curitiba, PR \\ E-mail: danidurski@gmail.com
}

\begin{abstract}
Resumo: $O$ objetivo da pesquisa é o de propor e estudar diversos cenários envolvendo a localização das escolas municipais de São Jerônimo da Serra, a fim de minimizar as distâncias percorridas pelos estudantes até suas instituições de ensino. Na primeira etapa, realiza-se uma análise da atual localização das escolas municipais e dos dados do transporte escolar. Na segunda, são realizadas simulações considerando a localização das escolas existentes. Nessa etapa, são utilizados os algoritmos de Teitz \& Bart e Simulated Annealing para resolver o problema de p-medianas. Na terceira etapa, é realizada a alocação dos estudantes para as escolas mais próximas utilizando o algoritmo de Gillett \& Johnson modificado. Na última etapa, é aplicado o algoritmo Location Based Heuristic Adaptado para o roteamento do transporte escolar, com a finalidade de comparar os resultados obtidos com a situação atual do município.
\end{abstract}

Palavras-chave: Localização, Escolas, p-medianas.

\section{Introdução}

O presente trabalho apresenta um estudo para encontrar a localização ideal de novas escolas e distribuir as vagas para os alunos que moram mais próximos a elas. Essa pesquisa foi solicitada pela secretaria de educação do município de São Jerônimo da Serra, localizada no estado do Paraná, cuja intenção é construir uma escola com capacidade de 800 alunos. A cidade possui 11.337 habitantes (CENSO 2010) e tem 49,93\% da população vivendo em área urbana e o restante na área rural. A taxa de crescimento populacional é de $-3,52 \%$.

Foram utilizados os dados do CENSO 2010 (IBGE, 2013), da Secretaria de Estado do Desenvolvimento Urbano (SEDU) e da Secretaria de Estado da Educação (SEED), para a realização deste trabalho. Esses dados foram processados pelo software geográfico Arcview para que conversões e adaptações fossem realizadas e então aplicadas meta-heurísticas para a obtenção das localizações, alocações e das rotas do transporte escolar.

O problema de localização de facilidades é caracterizado como o modelo de pmedianas, que visa minimizar todas as distâncias dos pontos de demanda (aluno) até a mediana (escola) mais próxima. Esse modelo é dito NP-Hard (PIRES, 2002), pois o número de soluções cresce exponencialmente com o aumento do número de vértices. Nesse aspecto torna-se necessário o uso de técnicas aproximadas para a resolução do mesmo.

A pesquisa está dividida em encontrar a melhor localização das escolas, considerando as já existentes, distribuir os alunos para elas e realizar o roteamento escolar. Sendo assim, optou-se por aplicar o Algoritmo de Teitz \& Bart e o Simulated Annealing para o problema de localização de escolas, o algoritmo de Gillett \& Johnson para o problema de alocação e finalmente o Location Based Heuristic Adaptado, para o problema do transporte escolar.

\section{Revisão de Literatura}

No Brasil, existem vários trabalhos relacionados com o estudo da localização de escolas. Pizzolato et al. (2004) realizam uma síntese de algumas linhas de experiência no 
Brasil, onde mostra e compara estudos de localizações de escolas em Nova Iguaçu, Nilópolis, Niterói, Ilha do Governador e Fortaleza. Pires (2002) realiza sua pesquisa na cidade de Coronel Vivida - PR. Os vértices utilizados são de 63 prédios de escolas, sendo que apenas 14 dessas estão ativas. Para a determinação dos prédios a serem utilizados é utilizado o Algoritmo Genético e para designar os estudantes até as escolas é utilizado o algoritmo de Gillett \& Jonhson.

Silva e Pizzolato (1996) realizam uma avaliação da Localização da Rede de Ensino Público de Niterói - RJ. Primeiro comparam a demanda com a oferta de vagas de acordo com os dados do censo e da secretaria de educação. Depois avaliam a situação atual e propõem a localização ideal das escolas no município. Barcelos, Pizzolato e Lorena (2004) resolvem o problema de localização utilizando os problemas de p-medianas capacitados e não-capacitados na cidade de Vitória - ES. os autores avaliam a situação atual com o auxílio do Sistema de Informações Geográficos (SIG) Arcview. Menezes, Diallo e Pizzolato (2011), os autores apresentam o estudo sobre o planejamento da rede escolar de Guaratiba, Rio de Janeiro, considerando a previsão de crescimento populacional para os anos de 2015 e 2020. Para a resolução do problema foi utilizada a ferramenta de otimização AIMMS, encontrando a localização das novas escolas usando os modelos matemáticos de PMC e o PLMC.

\section{Materiais e Métodos}

Para a resolução do problema real, as escolas já existentes são fixadas como medianas e as técnicas são aplicadas somente para a quantidade de medianas a serem encontradas.

\subsection{Teitz \& Bart}

O algoritmo apresentado em Teitz \& Bart (1968) é um clássico das heurísticas destinadas a solução de p-medianas. Esse é um método simples e de fácil implementação. Sua técnica baseia-se em quatro passos. O primeiro é construir uma solução inicial, o segundo rotular todos os vértices que não são medianas como "não analisados". O terceiro, a partir da solução atual, trocar todas as medianas, uma de cada vez, por um vértice candidato, analisar o custo e escolher a melhor solução. Uma iteração acaba quando todos os vértices não analisados forem testados. Se durante uma iteração não houver modificação na solução atual, o algoritmo é finalizado, e é apresentada a solução atual como solução do problema de p-medianas..

\subsection{Simulated Annealing}

Essa técnica é inspirada no trabalho de Metropolis et al. (1953) que apresentam um processo físico denominado Annealing que consiste em aquecer em uma caldeira um sólido (metais, vidro, etc.) até seu derretimento e depois esfriar lentamente de modo que obtenha a mínima energia do sólido. Isso acontece, pois quando um sólido é submetido a altas temperaturas, as moléculas podem mover-se livremente e após o seu resfriamento suficientemente lento obtém-se estruturas rígidas e perfeitas com uma energia mínima.

Trinta anos depois, Kirpatrick, Gelatt e Vecchi (1983) publicaram suas pesquisas baseadas no processo descrito acima. Nesses trabalhos a técnica chamada de Simulated Annealing implementada para computadores faz uma analogia ao processo de Annealing e é aplicada para problemas combinatórios. Os estados possíveis pelos quais o material passa, correspondem às soluções do espaço de busca e a energia em cada um desses estados corresponde ao valor da função objetivo. Se energia mínima é encontrada, significa que chegou-se a uma solução ótima local ou global. A cada iteração, um novo estado é gerado a partir do anterior por uma modificação aleatória e calcula-se a nova energia.

\subsection{Gillett \& Johnson Modificado}

O algoritmo de designação proposto por Gillett \& Johnson Modificado (Corrêa, 2000) visa formar agrupamentos em torno de pontos de demanda pré definidos e com capacidades conhecidas.

Inicialmente considera-se todos os alunos sem designação. Sejam $\mathrm{L}_{1}(\mathrm{i})$ e $\mathrm{L}_{2}(\mathrm{i})$ a primeira e a segunda distâncias das escolas mais próximas do aluno i, respectivamente. Para 
cada ponto $\mathrm{i}$, é calculada a diferença d(i) $=\mathrm{L}_{2}(\mathrm{i})-\mathrm{L}_{1}(\mathrm{i})$ e organizadas em uma lista em ordem decrescente. A designação começa obedecendo a ordem da lista criada, ou seja, os pontos i que possuem maior diferença são designados primeiro, a designação segue até que uma capacidade seja esgotada. Toda vez que uma capacidade for esgotada, e ainda houver alunos a serem designados, a diferença é recalculada e o processo é refeito. $\mathrm{O}$ algoritmo acaba quando todos os alunos forem designados.

\subsection{Location Based Heuristic Adaptado}

O sistema de gestão do transporte (SIGET) do estado do Paraná, utiliza a heurística proposta no trabalho de Siqueira e Souza (2012) na roteirização do transporte escolar. O algoritmo é aplicado nas cidades do estado do Paraná pela rede municipal e estadual de ensino contemplando os estudantes do ensino fundamental e médio transportando-os de pontos próximos de suas residências até as escolas em seus períodos de estudo.

O algoritmo que calcula as rotas do transporte escolar é dividido em três etapas: determinação dos pontos de parada dos veículos, cálculo das distâncias entre os pontos de parada até as escolas e determinação das rotas. Os dados utilizados são fornecidos pela SEED e pela SEDU, referentes ao $1^{\circ}$ semestre de 2013.

\section{Resultados e Conclusões}

A fim de apresentar uma solução para a resolução do problema apresentado pela secretaria de educação do município, foram criados alguns cenários e realizados testes para encontrar qual a melhor solução para a cidade. A seguir são descritos 4 casos e com seus resultados mostrados nas figuras 1, 2, 3 e 4, e comparados nas tabelas 1 e 2 . Os resultados nas figuras podem ser observados de acordo com a cor da bolinha, que indicam os alunos, que foram designados para as escolas de mesma cor, indicadas pelos triângulos.

\section{Caso 1: Previsão de construção de uma nova escola municipal}

Nesta análise, as 10 escolas municipais existentes permanecem em suas localizações com suas capacidades, e é considerada a criação de uma nova escola municipal.

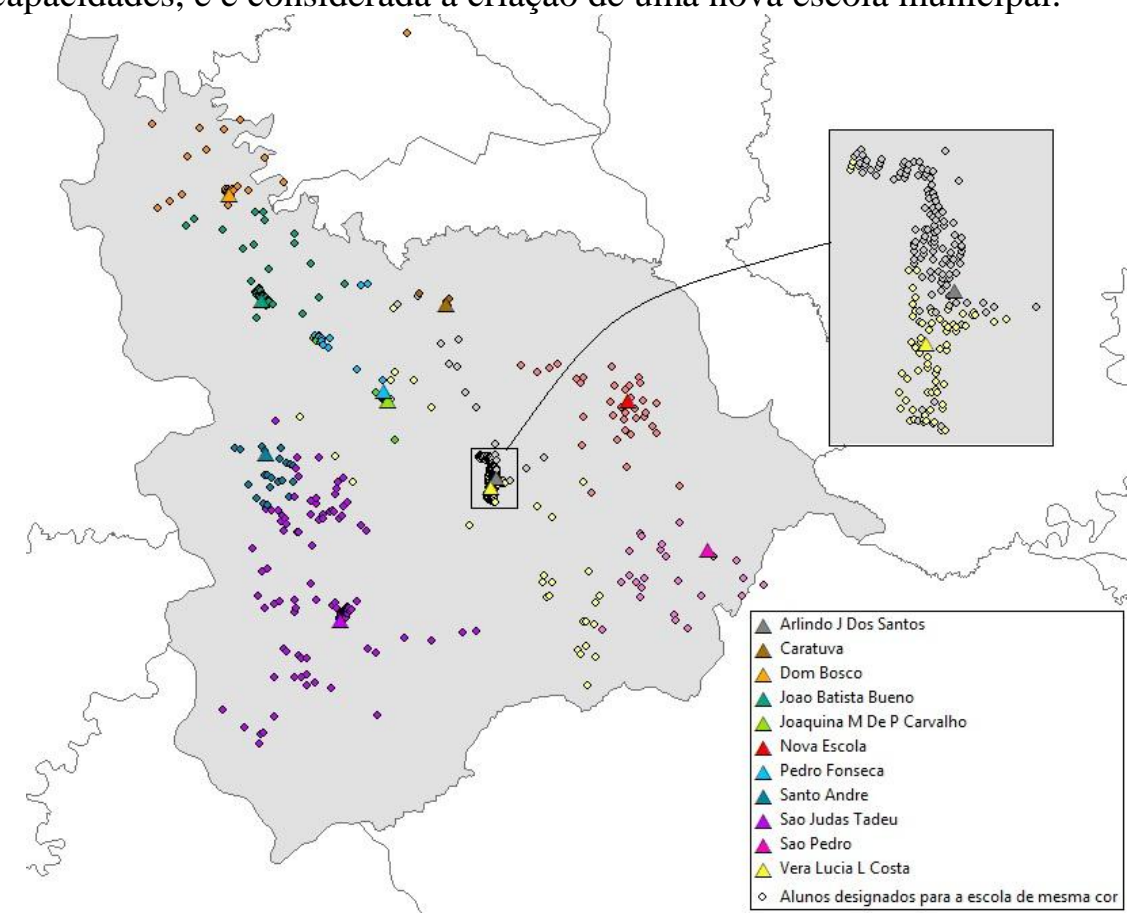

Figura 1: Resultado do Caso 1 - Escolas municipais e os alunos com suas devidas designações

\section{Caso 2: Previsão de construção de duas novas escolas municipais}

Esse quadro é similar ao caso 1, uma vez que considera-se os colégio existentes no município, é efetuada a alocação dos estudantes, e é calculado o custo do transporte escolar, a 
diferença é que no anterior foi proposta a criação de uma nova escola e neste caso foram propostas duas novas escolas.

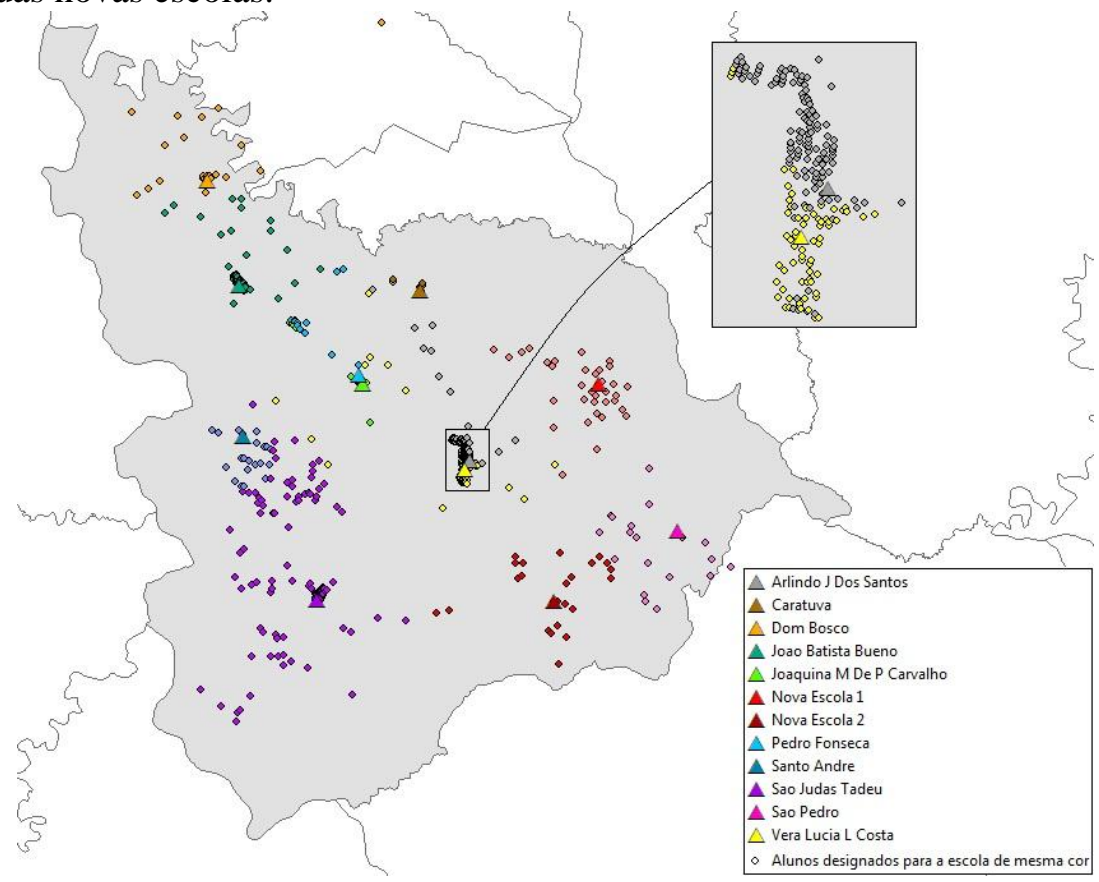

Figura 2: Resultado do Caso 2 - Escolas municipais e os alunos com suas devidas designações

\section{Caso 3: Aumenta-se a capacidade de duas escolas municipais}

Neste contexto não é criada uma nova escola, trata-se da melhor utilização dos prédios em que encontram-se as escolas Arlindo J. dos Santos e Prof. Vera Lúcia Costa, pois nos dois casos essas instituições dividem o prédio com as escolas, que hipoteticamente cedem o espaço que ocupam para as escolas municipais. Portanto, é considerado um aumento no número de vagas dessas duas escolas, e as demais permanecem sem alterações.

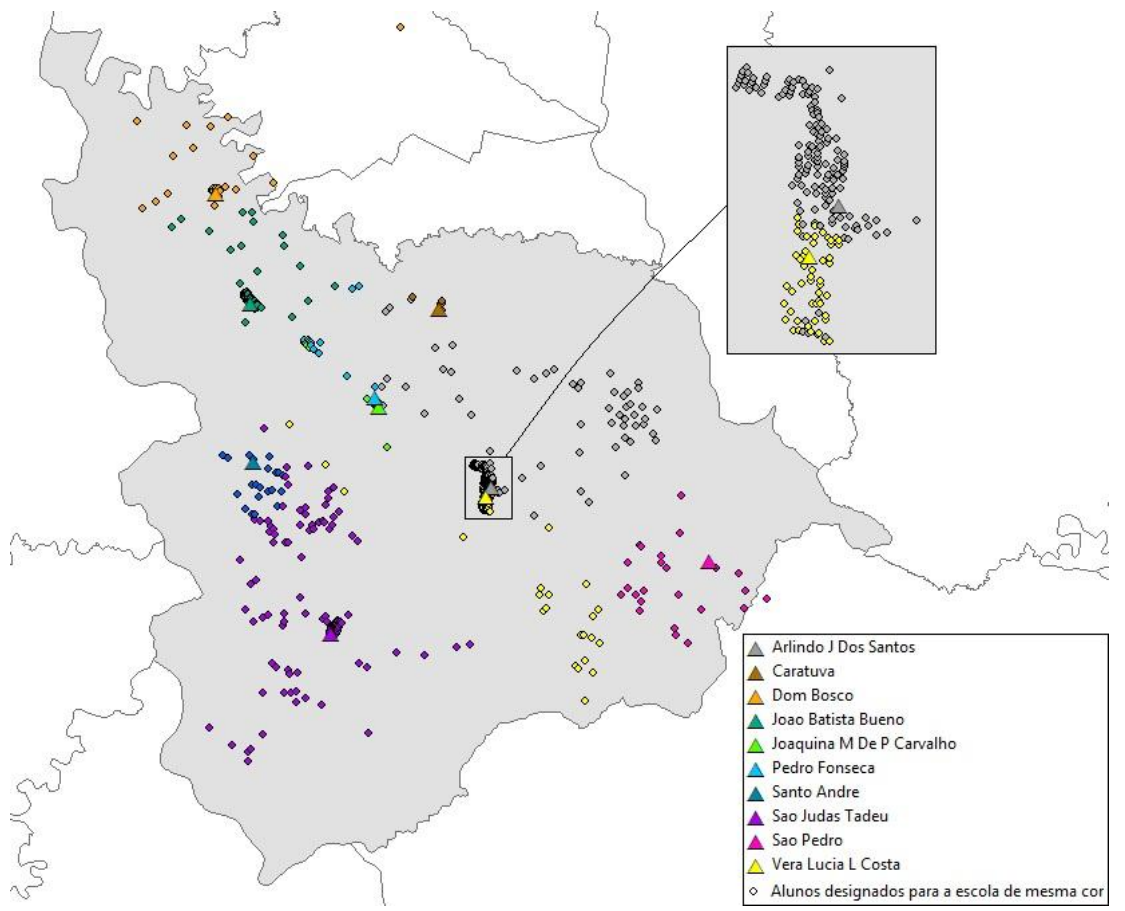

Figura 3: Resultado do Caso 3 - Escolas municipais e os alunos com suas devidas designações 
Caso 4: Aumenta-se a capacidade da escola Prof. Vera Lúcia Costa e propõe-se uma nova instituição

Nessa simulação, das 10 escolas existentes, uma deixa de existir e em outra tem sua capacidade aumentada, as demais permanecem sem mudanças. A escola municipal Arlindo J. dos Santos que divide o prédio com uma escola estadual, deixa de existir, devolvendo o espaço que é de propriedade do estado. O colégio municipal Prof. Vera Lúcia Costa que trabalha em dualidade com outra escola estadual, passa a ocupar o imóvel por completo, que é de propriedade municipal, aumentando assim a capacidade da escola. Realizadas essas modificações, ainda propõe-se a construção de uma nova escola.

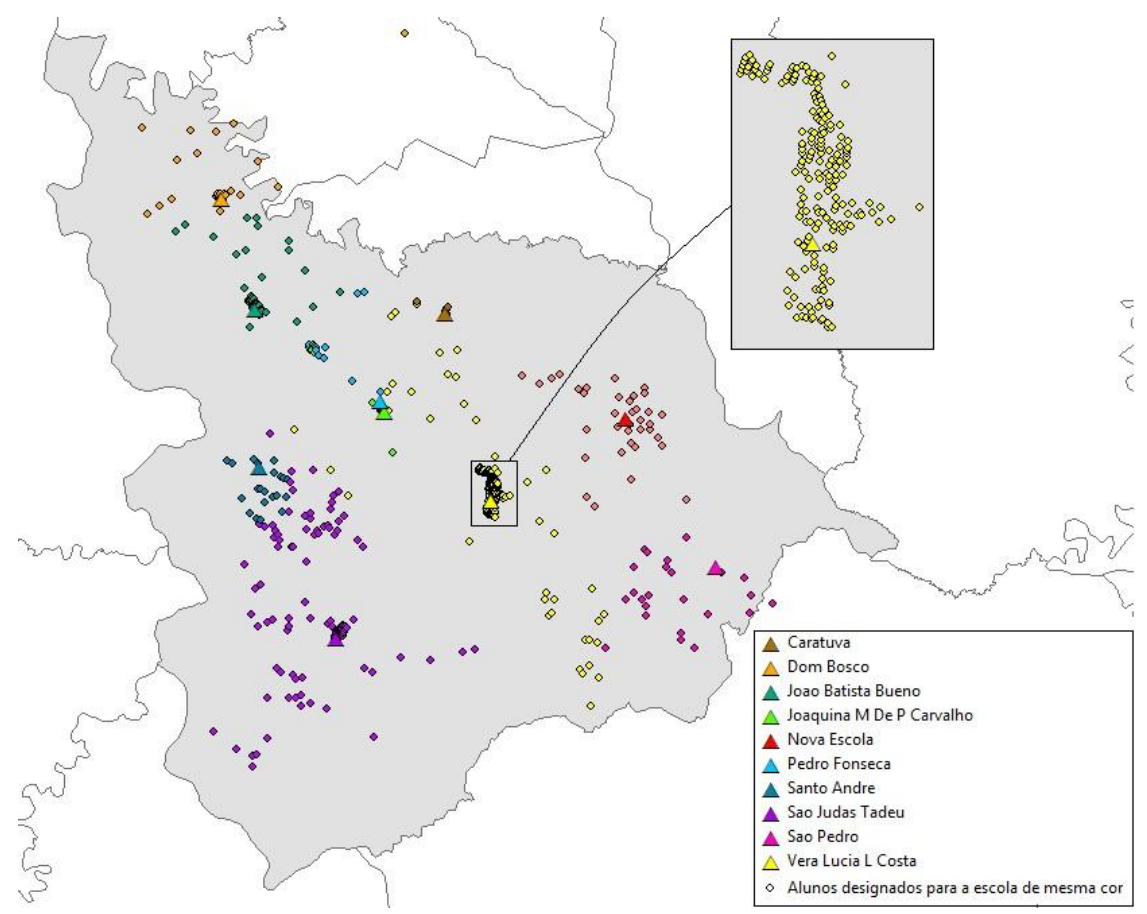

Figura 4: Resultado do Caso 4 - Escolas municipais e os alunos com suas devidas designações

\begin{tabular}{l|ccc}
\hline & $\begin{array}{c}\text { Distância total } \\
(\boldsymbol{k m})\end{array}$ & $\begin{array}{c}\text { Distância média por aluno } \\
(\mathbf{k m})\end{array}$ & $\begin{array}{c}\text { Tempo computacional } \\
(\mathbf{s e g})\end{array}$ \\
\hline Caso 1 & 1.647 & 1,9 & 0,20 \\
Caso 2 & 1.470 & 1,7 & 0,17 \\
Caso 3 & 1.896 & 2,2 & 0,21 \\
Caso 4 & 1.745 & 2,1 & 0,15 \\
\hline
\end{tabular}

Tabela 1: Comparação das distâncias obtidas pelo algoritmo de Gillett \& Johnson Modificado

\begin{tabular}{l|llllll}
\hline & & Veículos & Distância $(\boldsymbol{k m})$ & $\begin{array}{l}\text { Alunos } \\
\text { atendidos }\end{array}$ & $\begin{array}{l}\text { Alunos } \\
\text { próximos das escolas }\end{array}$ & $\begin{array}{l}\text { Alunos } \\
\text { que não usam TE }\end{array}$ \\
\hline Caso & Manhã & 20 & 1398,7 & 344 & 86 & 501 \\
atual & Tarde & 20 & 1472,6 & 308 & 116 & 564 \\
\hline Caso 1 & Manhã & 20 & 1360,6 & 333 & 108 & 491 \\
& Tarde & 20 & 1415,1 & 264 & 126 & 558 \\
\hline Caso 2 & Manhã & 20 & 1368,0 & 313 & 117 & 491 \\
& Tarde & 20 & 1444,5 & 261 & 126 & 558 \\
\hline Caso 3 & Manhã & 20 & 1422,9 & 336 & 95 & 491 \\
& Tarde & 20 & 1411,7 & 269 & 117 & 558 \\
\hline Caso 4 & Manhã & 20 & 1314,0 & 280 & 101 & 491 \\
\hline
\end{tabular}




$\begin{array}{llllll}\text { Tarde } & 19 & 1352,4 & 306 & 120 & 558\end{array}$

Tabela 2: Comparação dos dados do Transporte Escolar

Como é visto na tabela 2, em todos os casos, além de haver uma redução na quilometragem total das rotas do transporte escolar, os resultados da pesquisa mostram um aumento no número de estudantes que moram próximos de suas escolas, podendo deixar de utilizar o transporte escolar.

Com base nas pesquisas realizadas, constatou-se que a construção de uma nova escola na cidade, contribui na redução das distâncias percorridas pelos alunos até as escolas e também na quilometragem total do transporte escolar, mas através dos resultados obtidos, conclui-se que não há necessidade da construção de uma escola para 800 alunos como foi solicitado pela secretaria de educação do município, pois com a designação realizada observou-se que as vagas das escolas propostas não são preenchidas nem na metade da capacidade requerida pela secretaria do município.

\section{Referências}

[1]BARCELOS, F. B.; PIZZOLATO, N. D.; LORENA, L. A. Localização de escolas do ensino fundamental com modelos capacitados e não-capacitados: caso de Vitória/ES. Pesquisa Operacional, v. 24, p. 133-149, 2004.

[2]CORRÊA, E. S. Algoritmos genéticos e busca tabu aplicados ao problema das p -medianas. Dissertação (Mestrado) - UFPR, Curitiba, 2000.

[3]IBGE. Instituto Brasileiro de Geografia e Estatística. 2013. Disponível em: <http://www.ibge.gov.br>. Acesso em: 15/07/2013.

[4]KIRPATRICK, S.; GELATT, C.; VECCHI, M. Optimization by simulated annealing. Science, v. 220, p. 671-680, 1983.

[5]MENEZES, R.; DIALLO, M.; PIZZOLATO, N. D. Escolas públicas em Guaratiba, Rio de Janeiro: aplicação de modelo da p-mediana capacitado e de máxima cobertura. XLIII SBPO, 2011.

[6]METROPOLIS, N. et al. Equations of state calculations by fast computing machines. Jounal of Chemical Physics, n. 21, p. 1087-1091, 1953.

[7]PIRES, L. A. Algoritmo genético aplicado na localização de escolas do município de Coronel Vivida - PR. Dissertação (Mestrado) - UFPR, Curitiba, 2002.

[8]PIZZOLATO, N. D. et al. Localização de escolas públicas: síntese de algumas linhas de experiência no Brasil. Pesquisa Operacional, v. 24, p. 111-131, 2004.

[9]SEDU. Secretaria Estadual de Desenvolvimento Urbano do Estado do Paraná. 2013. Disponível em: <http://www.paranacidade.org.br>. Acesso em: 10/06/2013.

[10]SILVA, G. G.; PIZZOLATO, N. D. Avaliação gerencial da localização da rede de ensino público de Niterói - RJ. Aval. Pol. públ. Educ., v. 4, n. 11, p. 129-142, 1996.

[11]SIQUEIRA, P.; SOUZA, L. Métodos heurísticos aplicados na construção de roteiros de transporte escolar para o estado do Paraná. Transportes, v. 20, n. 3, 2012. ISSN 2237-1346.

[12]TEITZ, M.; BART, P. Heuristics methods for estimating the generalized vertex median of a weighted graph. Operations Research, v. 16, n. 5, 1968. 